\title{
Synergy Model of Artificial Intelligence and Augmented Reality in the Processes of Exploitation of Energy
} \section{Systems}

\author{
Tomasz Wójcicki \\ Institute For Sustainable Technologies, National Research Institute, Radom 26-600, Poland
}

Received: May 27, 2015 / Accepted: July 14, 2015 / Published: February 29, 2016.

\begin{abstract}
The article presents a fragment of research and development, which objective was to develop technical tools and methodology to improve exploitation processes of energy systems. The author's model includes synergy of artificial intelligence and augmented reality. This solution, which combines modern technologies in order to improve the activities related to the continuity of energy supply, and reduce costs associated with the time needed to carry out exploitation activities and employment of qualified staff, is presented. This paper presents both theoretical foundations as well as the development of technical systems. The characteristics of exploitation processes of energy systems and possible technical conditions, as well as factors characterizing them, are discussed. The physical and software structures of the system and individual modules, as well as dependencies connecting them are demonstrated. The dependencies between physical and logical elements during the exploitation processes of energy systems, that determine decisions related to the evaluation of technical states and related activities are described. The advantages and limitations of the developed model which connects methods of data processing and analysis, interactive visualization processes and possible areas of application are as well discussed in detailed.
\end{abstract}

Key words: Energy system, exploitation, artificial intelligence, augmented reality, artificial neural networks.

\section{Introduction}

Exploitation of energy systems is an important issue related to the economy, and ensuring energy supplies to production facilities and households. Therefore, it is appropriate to develop new technical tools and methods that support this type of processes. The exploitation of such systems involves small, medium and large companies which, depending on the type of business, are able to draw energy from existing networks, as well as having the ability to produce it themselves with the use of various types of equipment. Machines and equipment for the processing or production of energy require regular inspection and repairs in order to ensure continuous functioning. This facilitates the maximization of time for the use of

Corresponding author: Tomasz Wójcicki, Ph.D., senior lecturer, research fields: artificial intelligence systems, exploitation and diagnosis of power systems. individual parts and optimization of their exchange terms, which translates into measurable cost savings, and the improvement of the financial condition of the involved enterprises. Due to great diversity and complexity of energy systems, the streamlining of the processes of monitoring and support of personnel during exploitation is problematic as a result of existence of a multilayered structure [1]. One of the factors that can contribute to the improvement of this situation is the compilation of information, which depends on the specific exploitation strategy that is developed. Progress in the area of data processing enables the development of increasingly sophisticated computer diagnostic techniques which allow for the efficient analysis of signals, while at the same time, eliminating the human factor, which facilitates an increase in the objectivity of the results. Another important component of information systems is the 


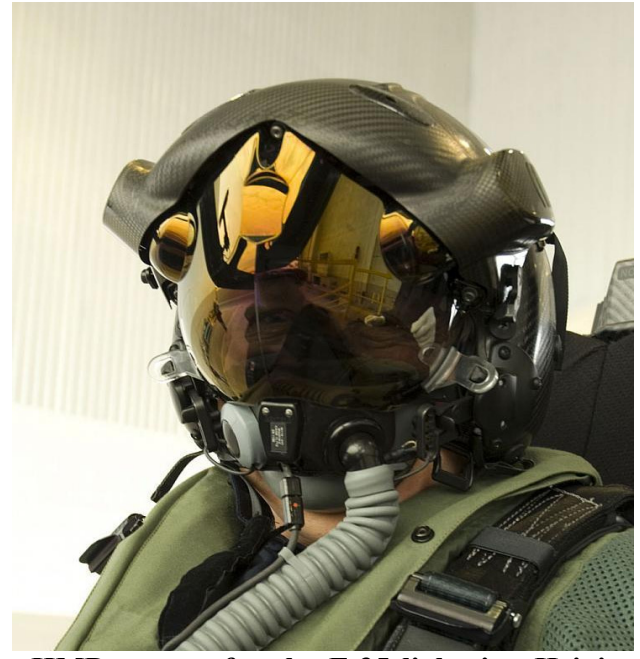

Fig. 1 HMD system for the F-35 lightning II joint strike fighter.

possibility of interactive visualization, which significantly improves the efficiency of ongoing activities. One promising technology is augmented reality-AR [2], which can have a significant impact on the processes of human-machine communication. This technology is characterized by the imaging of fragments of an actual environment, which is then enriched with the computer generated projection of virtual objects, and this complex visualization is then presented to a user of the system. The initial work on the use of this technology (which was not yet termed "augmented reality") was carried out in the 1960's, when the HMD (helmet mounted display) display was produced [3]. An example of the HMD system is shown in Fig. 1. The term "augmented reality" was used in the 1990's for the first time, by group of scientists working on a system for Boeing which supported the identification of wiring harnesses in the aircraft constructed by them. Currently, AR technology has a steadily increasing number of applications in more and more areas.

\section{Exploitation Processes of Energy Systems}

Exploitation is the set of all events, phenomena, and processes taking place in an actual object from the end of its production until liquidation [4]. The most important indicator of the quality of exploitation is exploitation economy, which is associated with the level use of exploitation potential contained in the analyzed object or system. During the use of technical objects, including energy systems, a key role is played by the methodology of operator handling (following the guidelines published in manuals, etc.). Therefore, all actions that are taken are relevant in relation to the object which is the subject of exploitation, including those that are organizational, technical, economic and social. Actions may be taken intuitively (to a large extent dependent on the acquired user's a-priori knowledge) or in accordance with accepted principles established by the system manufacturer. In order to maintain the potential exploitation of energy system components for the specified period of exploitation, and to ensure safe and economical use, it is necessary to perform non-operationally (performing periodic actions required by the manufacturer or implemented on the basis of an independent assessment of state). Lack of knowledge of the exploitation process may cause negative effects both for individual components as well as the entire system. Effective exploitation of energy system components requires that their users act in accordance with settled rules, which can be: prescriptive, propositional or critical. In the exploitation of technical objects, it is possible to isolate processes such as: use, diagnosis (including the forecasting of states), operation, management, acquisition, processing and presentation of data. The technical state of an object is determined by a set of values of characteristic attributes, and it is the result of actions and conditions in which the technical object was operated. This state is constantly changing, and among the changes are two basic states: the airworthiness state (object fulfills functions that have been set up for it), and the unfitness state (object does not fulfill functions that have been set up for it). In a number of publications, a third state is also described: partial unfitness (object does not fulfill all functions) [5]. Changes of technical state are associated with a number of processes in the technical object or in its 
surroundings, such as fatigue aging (variable loads that cause unwanted effects in the form of circuit burnout or deformation). The changes may be reversible or irreversible. They can also be classified into one of three basic groups: critical (causing a threat to the life and the health of users, as well as the natural environment), boundary (which can negatively affect the performance of machines), acceptable (which may affect the originally established method for using the object). Wear processes of energy systems are shown in Fig. 2.

If none of the attributes that characterize the technical state of the energy system element exceed the limit value, it is then considered that, the technical and operational characteristics are consistent with the assumptions made when it was designed and manufactured, and the elements of the energy system can perform all the tasks intended for it. Processes that cause wear can affect the efficiency of the individual elements of the system. The decrease in efficiency can be caused to a large extent by changes that occur during exploitation. They can affect the amount and frequency of the occurrence of defects, and cause permanent damage to energy system elements when they are not subjected to repair. Another specific factor for exploitation is that of operating states, which may include: active use, storage, long-term maintenance, constant maintenance, transfers, main repairs, indirect

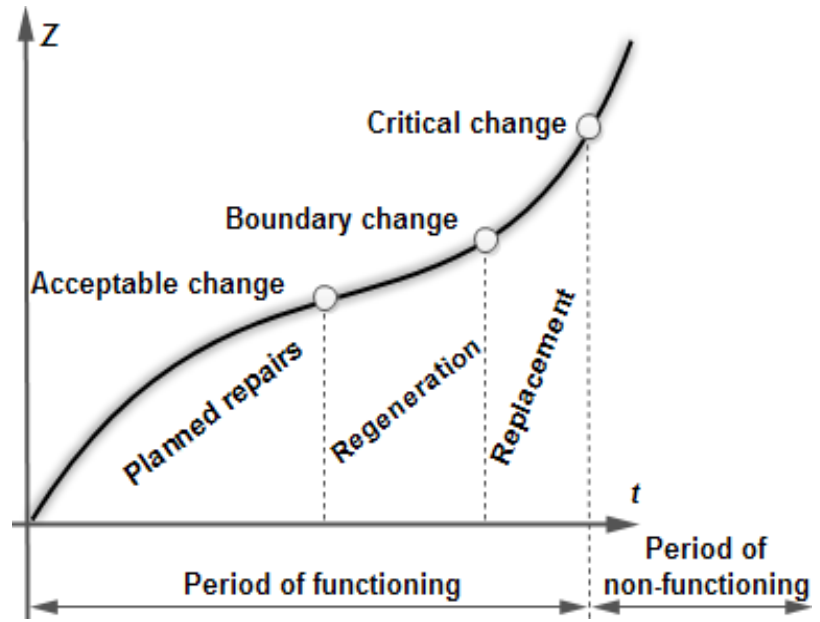

Fig. 2 Wear processes of energy systems: $Z$-wear degree, $t$-time. repairs, current repairs, ongoing maintenance, liquidation and transportation.

\section{Synergy Model of Artificial Intelligence and Augmented Reality}

The developed model solution is an exemplification of the objective of the work, which was to develop technical tools and methods for supporting technical staff in the works related to the exploitation of energy systems through the use of augmented reality technology and artificial intelligence. The general formal model which describes the process of synergistic support activities related to the exploitation of energy systems is as follows:

$$
F_{M}: X_{M} \rightarrow Y_{M}
$$

where, $F_{M}$-operator of the model, $X_{M}$-space of input values, $Y_{M}$-space of output values.

The space of input values $X_{M}$ creates discrete data which include: images recorded by cameras of AR system, the types of processes that will be carried out (diagnostics, repair, use) and information on the expected values of the parameters of energy system components. The space of output values $Y_{M}$ creates information on the measured values of parameters of energy system components and diagnosed technical states. With the participation of the operator of the model, the conversion of input values to output values, the use of interactive operational instructions (visual cues) and the participation of the person who operates the system, as well as computational models, is based, inter alia, on methods from the area of so-called artificial intelligence which is being utilized. The physical structure of the system consists of stationary and mobile parts (Fig. 3).

The main elements of the physical structure of the developed system are: server, projection device, a digital camera which records images analogous to that which the operator sees, the user's computer, a measurement system and a keypad. A characteristic feature of this system, the structure of which is 


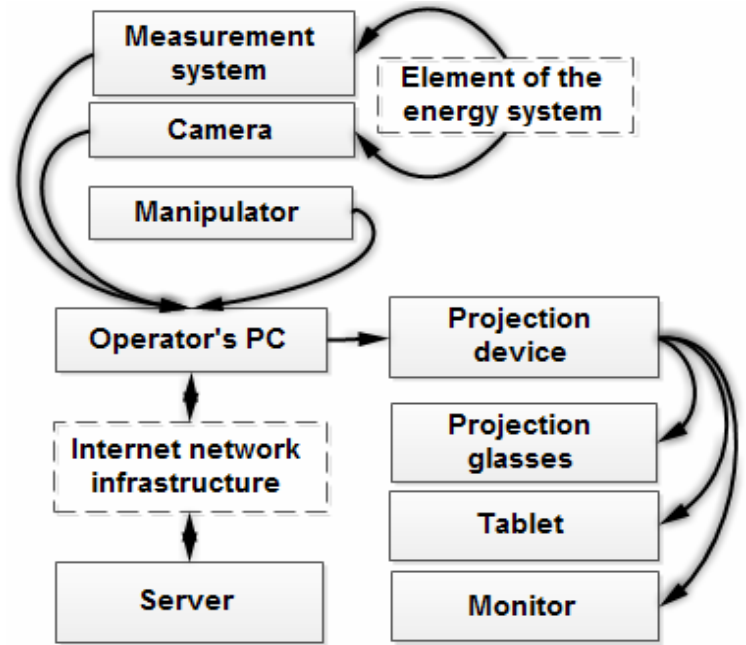

Fig. 3 Physical structure of the support system of energy systems.

presented in its mobility, which provides the user the ability to move around an area in which processes are carried out that are associated with diagnosis, repair or use of elements of energy systems. As a projection device, it is possible to use a computer monitor, tablet or projection glasses [6]. Device selection depends on the specifics of works planned for implementation. In case a tablet is used, it is also utilized as a user's personal computer. Projection glasses are effective, because the user has his hands free, and can smoothly carry out activities related to the diagnosis or repair of energy systems. The software structure of the system is shown in Fig. 4. The software of the system, analogous to the hardware, is comprised of two parts: stationary and mobile. The stationary system includes: an application server, data synchronization system, data analysis system, and a knowledge base coupled to the database. The Mobile system software consists of a presentation-communication platform functioning in a Flash environment [7], web browser and measuring system. The use of a Flash environment and a web browser provides an advantage because there is no need for an installation process for dedicated software, because it is automatically downloaded from the application server which is located in the stationary system.

An important aspect of the model related to diagnostics is the data analysis system, which is based on the use of artificial intelligence mechanisms. At this point, a number of computational models can be utilized, such as fuzzy logic, genetic algorithms, probabilistic networks, etc. In the developed model system, artificial neural networks have been applied, the architectures of which are chosen from a defined set which depends on the analyzed problem. The basis of the network is a multi-layer perceptron [8] with a variable size of input and output vector, and a variable number of neurons in two hidden layers. This model

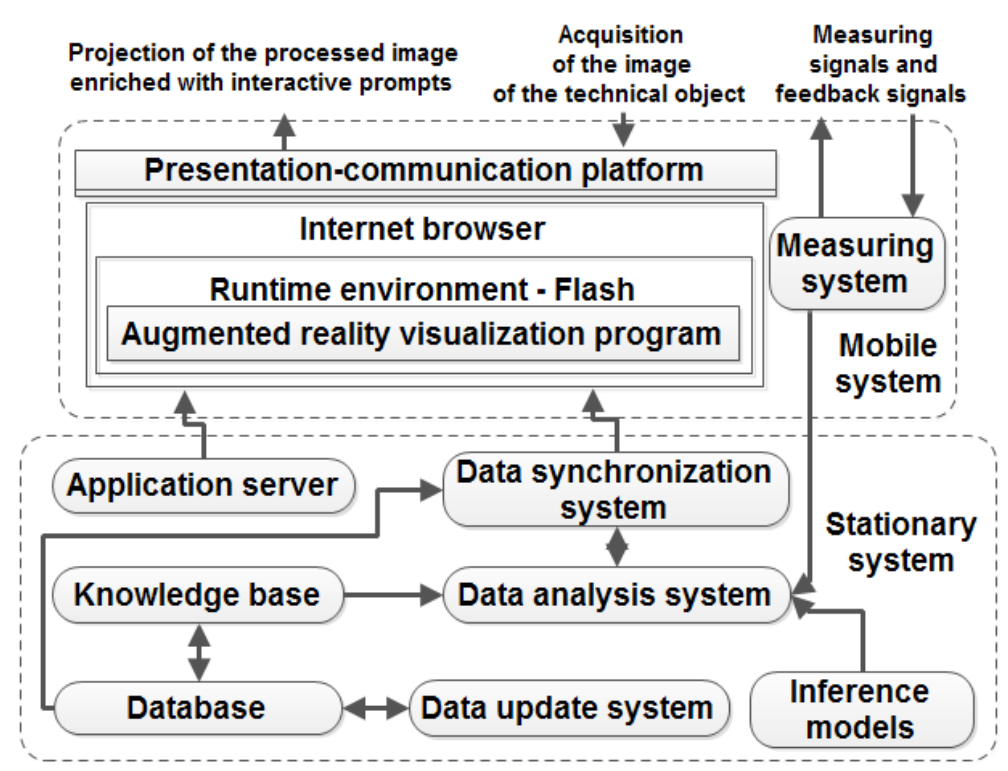

Fig. 4 Software structure of the support system of energy systems. 
was chosen because of the possibility of mapping different real systems. The use of two hidden layers influences the expansion of a set of possible reactions of networks that can be applied in complex problems (formal confirmation of this theory is the Kolmogorov theorem [9]). It was assumed that, the basis for the functioning of neurons would be a sigmoidal, unipolar activation function [10], which, because of its properties (smoothness, differentiability, monotonicity) is an effective model for calculating output values. Adaptation of synaptic weights of neural network structures for classification purposes (e.g., identification of electrical grid faults or an identification method of repair activities) takes place in cycles, according to Eq. (2):

$$
w_{i j}(g+1)=w_{i j}(g)+\Delta w_{i j}(g)
$$

where, $g$-learning cycle, $w_{i j}(g)$-previous synaptic weight connecting neurons $i$ and $j, w_{i j}(g+1)-$ new synaptic weight connecting neurons $i$ and $j$. The objective function during the learning process is a measure of error at the output of network, which is represented by the Eq. (3):

$$
E_{M S E}=\frac{1}{2} \sum_{k=1}^{R}\left(t_{k}-y_{k}\right)^{2}
$$

where, $R$-the size of output vector network, $k$-network output number, $t_{k}$-required value given in the learning sequence for the $k$-th output of network, $y_{k}$-value obtained for the $k$-th output of network.

The learning process leads to the mapping of relationships represented by the training set in network output vectors. The shape of the boundary decision in a multidimensional feature space depends on the weight modification of individual neurons, based on the data of the learning sequence. In order for the adjustment of weights for observation to be effective, it is necessary to provide sufficient input vectors during the learning process, due to the effect of the generalization of results.

Generated results are additional information for the system operator which supplies the video communication interface of the projection device, supporting the operator in carrying out activities related to the exploitation of energy systems. The steps of displaying operating instructions to the operator which supports the carrying out of exploitation processes may be presented with or without feedback from the data analysis system that processes the signals retrieved from the analyzed elements of energy systems. An important issue in the developed method which utilizes augmented reality is interaction between the projection of images enriched by the content of instructions containing the virtual objects (models 2D and 3D), and user and physical energy system elements (Fig. 5).

Operator evaluates the received image projection of energy system elements, enhanced by the content of instructions with added virtual objects, interprets their states, and decides what actions should be taken (Fig. 6).

Some of the activities carried out by the operator can cause the generation of confirmation signals received by the measurement system and present them in a visual way. These types of signals play a very important role in the improvement of the exploitation process, because they assure the operator of the correctness of

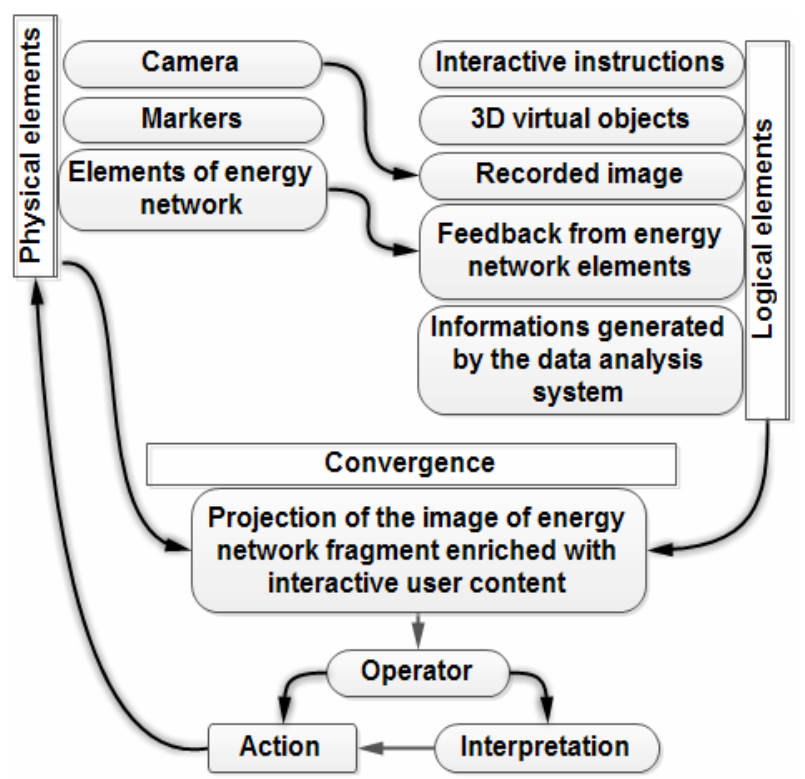

Fig. 5 Dependencies between physical and logical elements in the method of exploitation support of energy systems with the use of artificial intelligence and augmented reality. 


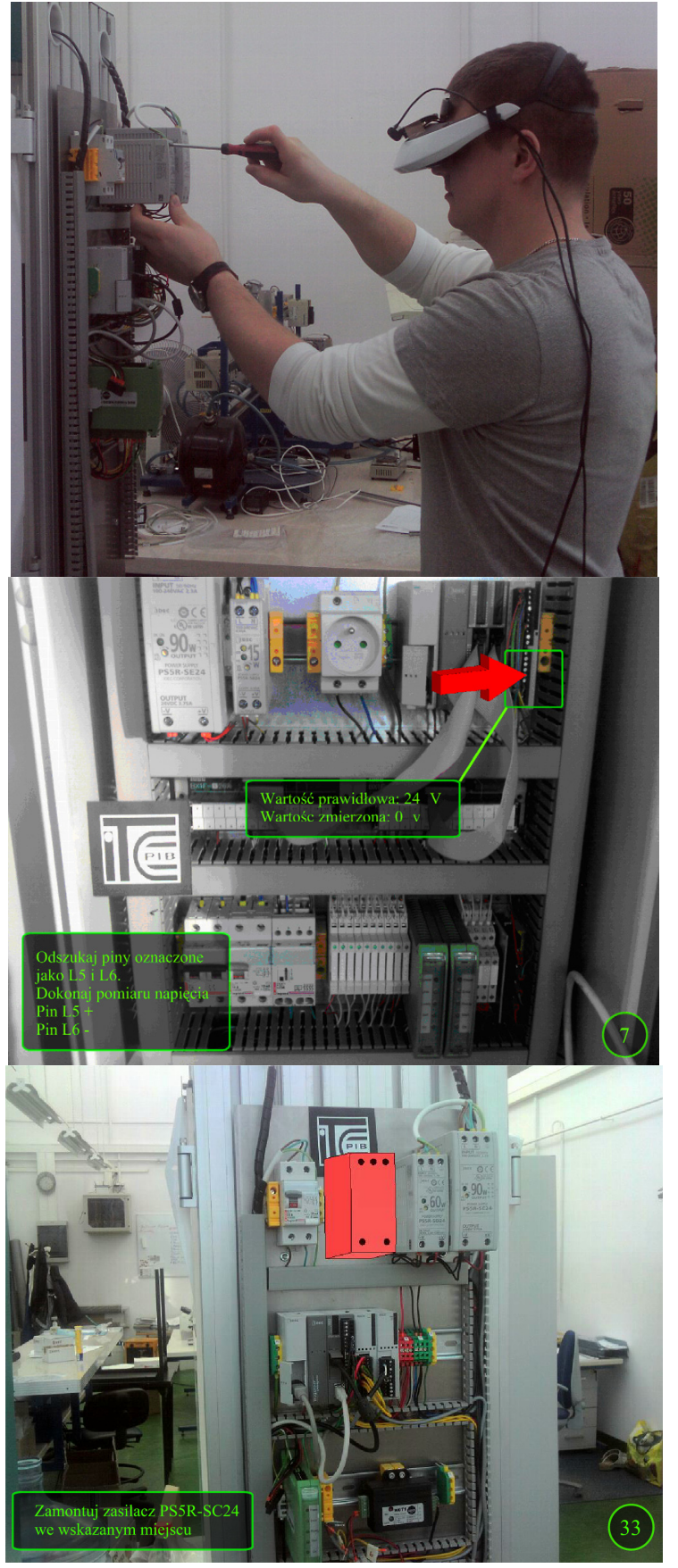

Fig. 6 Operator of a developed AR system, and the image seen by him during the processes related to the diagnosis of the energy system elements.

work that has been done. For activities that are not engaged in the generation of feedback, the correctness of performed actions is recognized by comparing the physical state of energy system elements, and visual presentation consisting of virtual objects by the operator.

\section{Results}

In order to confirm the suitability of the developed technical system and methodology to support the exploitation processes of energy systems, for fragment of energy system the studies were conducted. The fragment of energy system consisted of selected elements of a wind turbine (gearbox, power generator, control system, module of positioning of blades relative to the wind direction, braking system). These elements need to conduct periodic inspections and maintenance. To carry out activities related to the exploitation of these elements, six people that had never before led maintenance procedures for selected elements of wind turbines were selected. The group of people divided into two parts. The task of the first part of the group was to carry out selected exploitation activities only using instructions in classic form (paper). The second part of the group had developed technical system based on augmented reality technology. Individuals were asked to perform 23 operations. The aim of the study was to examine the impact of the use of the developed technical system and methodology by untrained staff on duration of exploitation works. The results are shown in Fig. 7.

It was found that, conducting works related to the exploitation of energy systems for people without

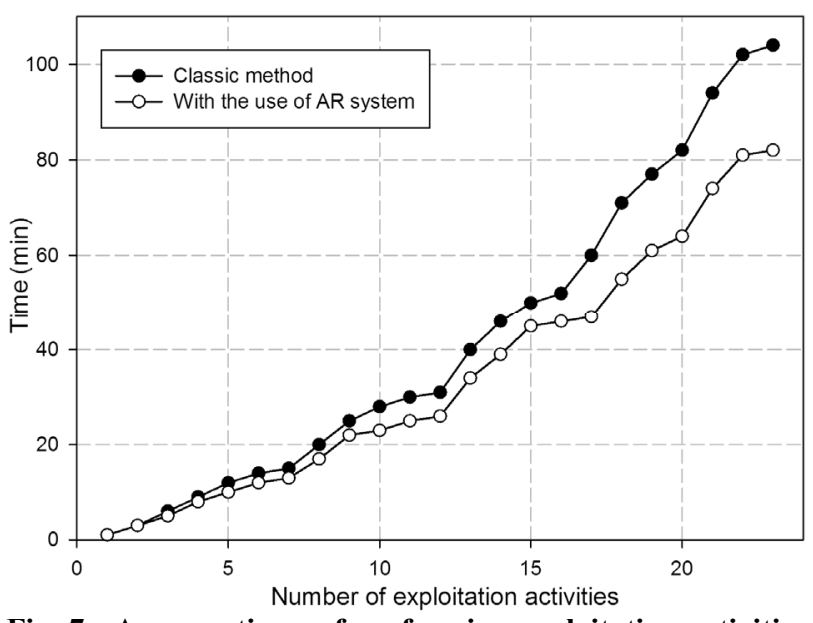

Fig. 7 Average times of performing exploitation activities for people using classic instructions (in paper form) and developed technical solution based on augmented reality technology. 
training is more effective in terms of time, for people who use the developed technical solution and methodology than for people who use only paper instructions. The time saving is greater if the exploitation process is more complex (requires a larger number of activities).

\section{Conclusions}

The author's developed model, which utilizes the synergy of elements of computational intelligence, and augmented reality improves work related to the exploitation of energy systems, which may include diagnostic processes, repair or maintenance. The use of modern information technology improves the effectiveness of these actions, through an interactive presentation of location of selected components of handled systems, presentation of diagnostic activities, descriptive presentation of suggestions in the form of text or graphics, presentation of location of measurement points and expected measurement values. Use of the proposed solution can significantly affect the shortening of diagnostic processes (in particular of energy system elements that are characterized by significant complexity), reduce costs associated with the training of technical personnel and reduce the size of the workforce by expanding the scope of work performed by individuals. Another advantage of the proposed solution is the reduction of the risk of accidents and the possibility of a more rapid restoration of energy systems to working condition. In actual conditions, it can have a significant impact on the reduction of financial losses associated with energy supply interruptions, and helps to ensure the continuity of production. The developed solution, in addition to the recognition of the state of energy system elements, can be used to conduct training of the technical staff (improvement of skills and competence required to perform works related to the exploitation of energy systems), which also illustrates the significant application potential of the described solution.

\section{References}

[1] Grigoras, G., Barbulescu, C., Cartina, G., and Comanescum, D. 2012. "Human Errors Monitoring in Electrical Transmission Networks." Journal of Energy and Power Engineering 6 (March): 425-8.

[2] Craig, A. 2013. Understanding Augmented Reality, Concepts and Applications. USA: Elsevier.

[3] Harding, T., Martin, J., and Rash, C. 2006. Helmet-Mounted Display Computer Model and Research Visualization Tool. USA: U.S. Army Aeromedical Research Laboratory.

[4] Mobley, R. 2004. Maintenance Fundamentals. USA: Elsevier Inc.

[5] Gołąbek, A. 1988. Operation and Reliability of the Machines. Warsaw: Warsaw University of Technology Publishing House.

[6] Kim, G. 2015. Human-Computer Interaction, Fundamentals and Practice. USA: CRC Press.

[7] Rapo, A., and Michael, A. 2013. Understanding Macromedia Flash 8 ActionScript2, Basic Techniques for Creatives. USA: Focal Press.

[8] Conan-Guez, B., and Rossi, F. 2004. "Multi-layer Perceptrons in Classification, Clustering, and Data Mining Applications." In Proceedings of the Meeting of the International Federation of Classification Societies (IFCS), 157-67.

[9] Li, M., and Vitanyi, P. 2008. An Introduction to Kolmogorov Complexity and Its Applications. USA: Springer.

[10] Aizenberg, I. 2011. Complex-Valued Neural Networks with Multi-valued Neurons. Berlin: Springer. 